\begin{tabular}{cc}
\hline International Journal of Engineering \& Technology, $7(2.8)(2018) 524-528$ \\
SPC & Website: www.sciencepubco.com/index.php/IJET \\
Research Paper & International Journal of Engineering \& Technology \\
\hline
\end{tabular}

\title{
Design and implementation of dimmer based smart street lighting system using raspberry Pi and IoT
}

\author{
Annareddy Sravani, P.Malarvezhi, R. Dayana \\ Department of ECE, SRM Institute of Science and Technology, Chennai, Tamil Nadu, India \\ *Corresponding Author Email: annareddysravani51@ gmail.com
}

\begin{abstract}
In olden days street lights were not operated in an automatic way. Automation of street lights has become apparent these days. But we can notice that we do not require high intensity light during night hours, i.e. when there is no traffic, no people in the streets or on roads and even in the early mornings. As per requirement, the light intensity can be reduced using dimmer circuit. Light dependent resistor (LDR) sensors are used to sense the darkness and Passive Infrared (PIR) sensors are to detect the objects. Raspberry Pi (Master node) and Arduino (Slave node) will communicate each other and they help the proposed system to work more effectively. Current sensor and Voltage sensor are used to measure the current and voltage respectively. By reducing the intensity at these times, energy can be saved to some extent and the data is uploaded to the cloud. We can monitor and control the street lights in a smart way as per our requirement. Fault detection, minimization of cost, reducing the loss of electricity and man power are also possible. Hence, this proposed smart street lighting system will be helpful to the society in cost effective way.
\end{abstract}

Keywords: Arduino, Cloud, Dimmer circuit, LDR and PIR sensors, Raspberry Pi.

\section{Introduction}

Power consumption of street lighting system is a major component in the India. As per global trends survey, we pay $18-38 \%$ of the energy bill for street lighting. We need to pay our attention on this so that we can look for better power efficiency for effective utilization of power and therefore our ultimate aim can be achieved i.e., saving power. Smart street lighting system is a simple but still a powerful concept. We may have observed that there are street lights still not OFF after 6:00a.m. Also, we can observe two things in some of the rural areas. There are no street lamps at all or no heavy traffic to use those facilities properly. Simply it concludes with the wastage of electricity.

Initially, the street lights were handled manually where in each of the street lamps, the control switch was set which was known as the first generation of the original street light. Alternate method that has been used was based on the optical control method in which the high-pressure sodium lamps were utilized.

A good street lighting system can provide safety, good visibility and comfort to vehicles/pedestrians to travel along the roads thereby lowering many malfunctions that cause all along the night and enhance the appearance of the locality. Contrarily, the design of poor lighting systems can result to indigent visibility and this will not be useful for vehicles or pedestrians going along the roads. Street lighting is designed poorly very frequently and has scanty maintenance (e.g., many failed lights are there), and adopts outdated technology for lighting, thus it consumes high energy and more money (due to street lights glowing during the day time), while often failing to provide reliable lighting System.

The explanation of high efficiency LED driver design has given by two control methods. Those are constant-voltage, current feedbacks respectively [1].
A survey on the cost analysis of smart lighting solutions for smart cities has given in [2]. The study of HTTP protocol and how it helps in communication between devices and user has been seen in [3]. In this work, the authors presented a fog-enabled IoT platform which is used to collect the data from sensors [4]. A dimmer circuit for various lighting devices, a review on triac controlled energy efficient dimmable led driver for street lighting was given in [5-7]. A discussion on hierarchically distributed Fog Computing architecture to support the integration of high number of infrastructure services and components in future smart cities was given in [8]. An energy-optimization control system for street lighting was presented. But the design and implementation cost is very high. Street Light Monitoring and Control System were implemented by integrating various sensors and controllers $[9,10]$. The paper named as Simple Stabilized Radio-Frequency Transfer with Optical Phase Actuation has described and demonstrated experimentally the function of a stabilized RF transfer technique [11]. The Seamless Key Agreement Framework for Mobile-Sink in IoT Based Cloud-Centric Secured Public Safety Sensor Networks paper explains that how to use IoT for better results [12]. IoT based smart grid communications and solution for the threats was explained in [13]. The IoT technologies for smart cities have been studied in [14].

Rest of the paper is arranged as follows, the existing system is discussed in section II. Section III enumerates the proposed system with respective block diagrams in detail. Section IV explains about the hardware components of this project. Section V discusses about the results of the proposed work. Section VI discusses the conclusion and future work. 


\section{Existing System}

Many of the places in India have the existing methods like noting the complaint, turning on/off the light manually. Generally, this is the time consuming \& needs manpower. The new method automatic ON/OFF and fault detection without human intervention is easier when compared to the existing system. We have many street lighting systems like brute-force search algorithm technique, solar street lighting but implementation and maintenance cost is high with these methods.

\section{Proposed System}

The proposed system consists of two nodes slave node and the master node namely. There are many slave nodes but master node will be only one and it controls all the slave nodes by passing commands to them. We connect slave node to every pole in the street like shown in figure 1. Here,we use Raspberry Pi as master node.

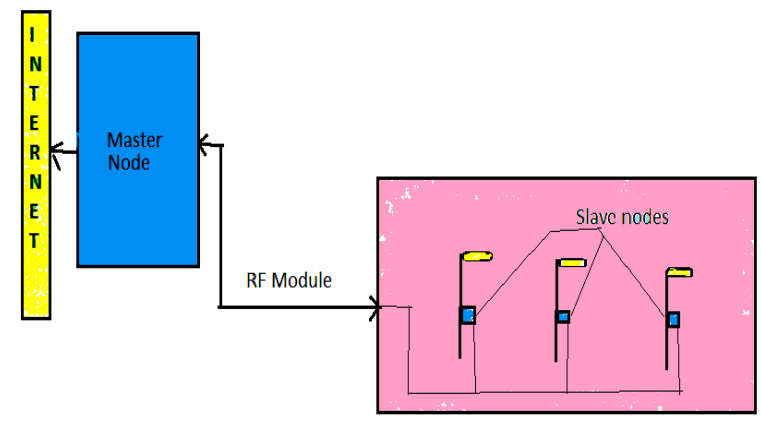

Fig. 1: Block Diagram of the proposed system

Arduino acts as a slave node and is shown in figure 2. Dimmer circuit is connected to slave for reducing the brightness of light according to our requirement. Generally, we don't need the light with full brightness all the time i.e., from 6:00 p.m to 6:00 a.m. In this project we fix the time that light glows with high intensity between 6:00 p.m to 11:00 p.m, from 11:01 p.m to 05:00 a.m light glows with medium intensity, from 05:01 a.m to 06:00 a.m light glows with low intensity. The rest of the time the light will be in OFF state. These all things will happen with PWM in the dimmer circuit. The above-mentioned timings are alright on normal days. In rainy season those timings are not suitable. So, we use LDR sensor. An LDR is a sensor that has a (variable) resistance and this will change with the light intensity that falls upon it. When there is no light, it will send the command to master that there is darkness and again the master will send a command like the light should turn $\mathrm{ON}$ now.

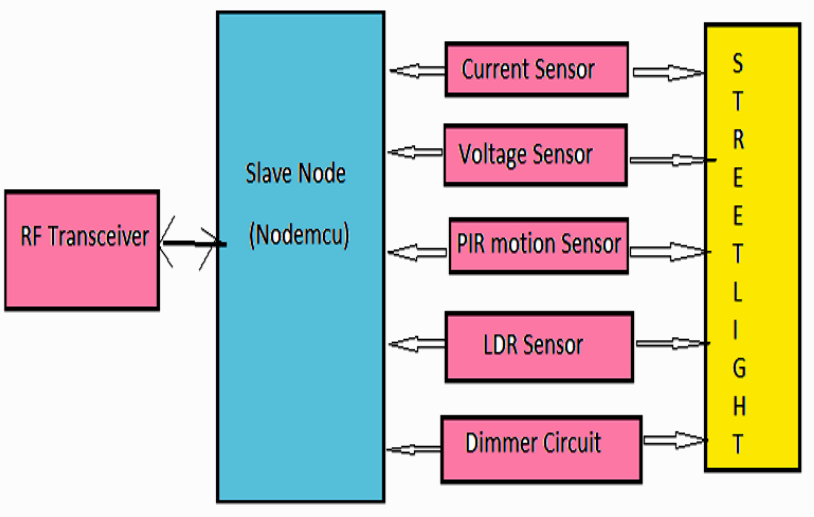

Fig. 2: Block diagram of the Slave node

PIR sensor detects objects and human. If anyone or anything comes near to the pole then it makes the light to glow with high intensity. RF transceiver is for communication between master and slave nodes. Current sensor measures the current. The flow chart shown in figure 3 tells the logic flow of the proposed project. The intensity of light changes as per given timings.

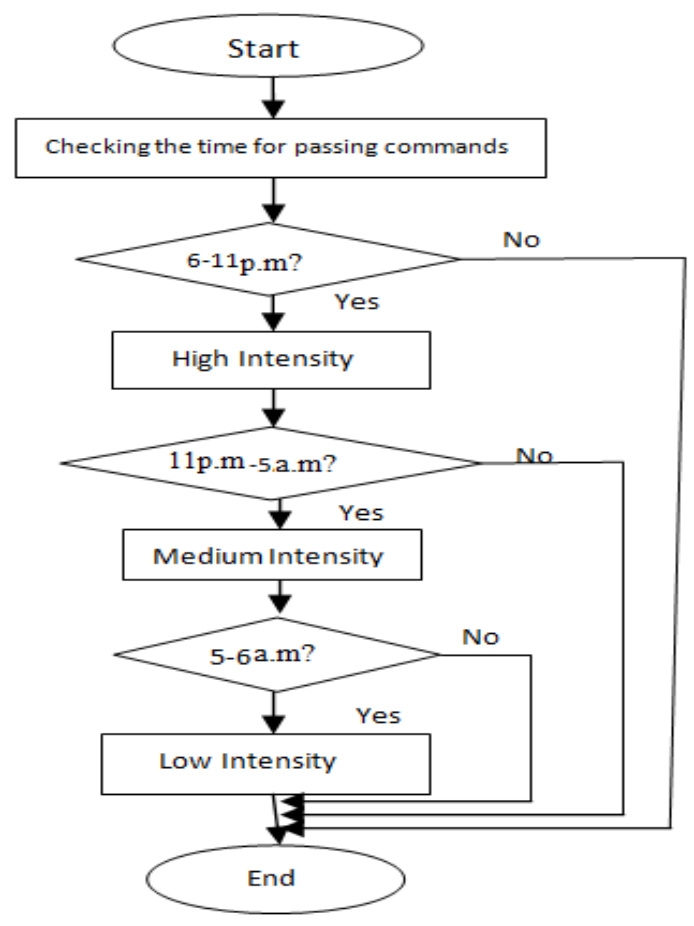

Fig. 3: Logic flow of changes in light intensity

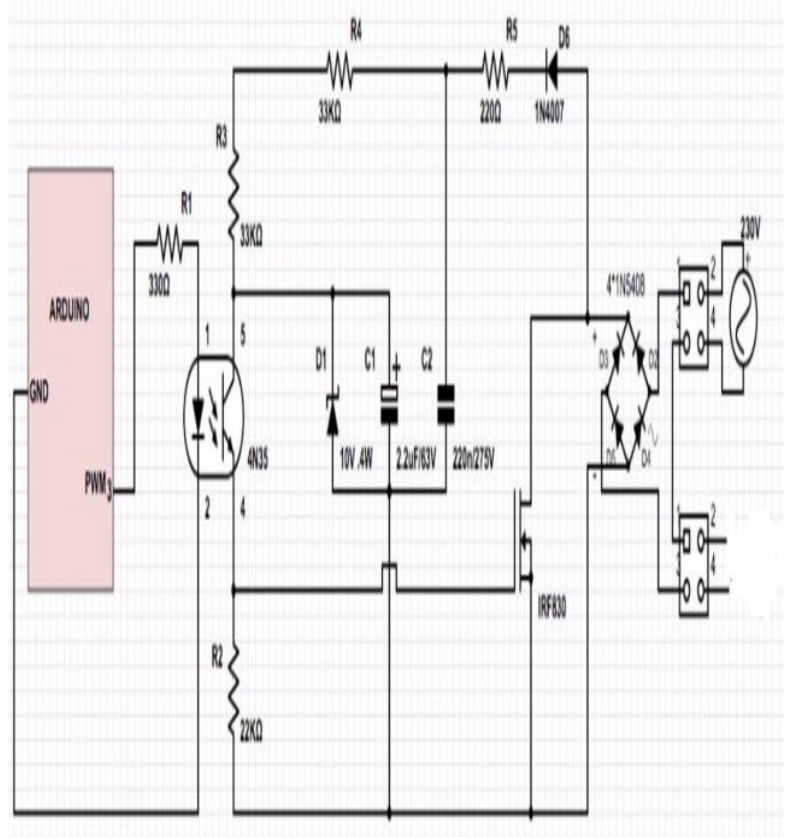

Fig. 4: Dimmer Circuit

A rectifier is formed by connecting the diode D6, the load resistor R5 and the capacitor C2. The resistor R5 is used to limit the current pulses passing through the diode D6 and it is shown clearly in figure 4 . The voltage across the capacitor $\mathrm{C} 2$ is regulated to a maximum value of $10 \mathrm{~V}$ by the resistors $\mathrm{R} 3$ and $\mathrm{R} 4$, the capacitor $\mathrm{C} 1$, and the diode $\mathrm{D} 1$. The gate is driven by the resistor $\mathrm{R} 2$ and the opto-coupler. The LED in the opto-coupler is protected by the resistor R1. Same as R5, R1 is also used to restrict the current so that we can apply the hard voltages securely.

Class-II isolation will be provided by the opto-coupler CNY65. This is good and safety of the regulator will be ensured. Transistor is connected to the positive terminal of the opto-coupler for 
MOSFET conduction. The rating of resistor R2 kept a bit high so we have to make the adjustments between the switching loss and the inductive voltages. This is basically performed to minimize the switching spikes as a result of parasitic inductances. Compare to PWM the MOSFET conducts for long duration. The voltage across the diode D1 will be equalized to $10 \mathrm{~V}$ over a $88 \%$ duty cycle at the time of voltage reduction across the MOSFET.

\section{Hardware Components}

Current sensor: ACS712 Current Sensor Module - 20A is based on ACS712 sensor, which will identify AC or DC current correctly and is shown in figure 5. The high AC or DC that will be identified will reach $20 \mathrm{~A}$, and the present current signal will be measured via input or output analog port of a microcontroller or an Arduino.

ACS712 Current Sensor Module - 20A Specifications:

1 . We can apply $4.5 \mathrm{~V}-5.5 \mathrm{~V}$ as supply voltage.

2 . The range of DC current measured is $20 \mathrm{~A}$.

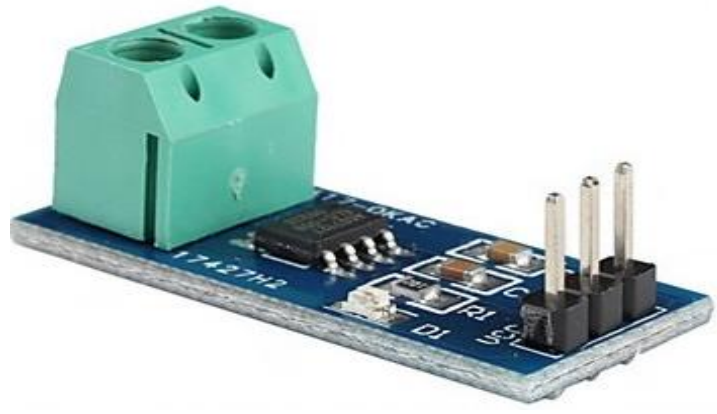

Fig. 5: ACS712-20A Current Sensor

LDR sensor: LDR sensor detects the light intensity. According to the light intensity, if there will be light then the LDR resistance will become low. If the light intensity is high then the resistance of LDR will become low. The sensitivity of the LDR towards the light will be adjusted with the help of potentiometer knob and the sensor is shown in figure 6 .

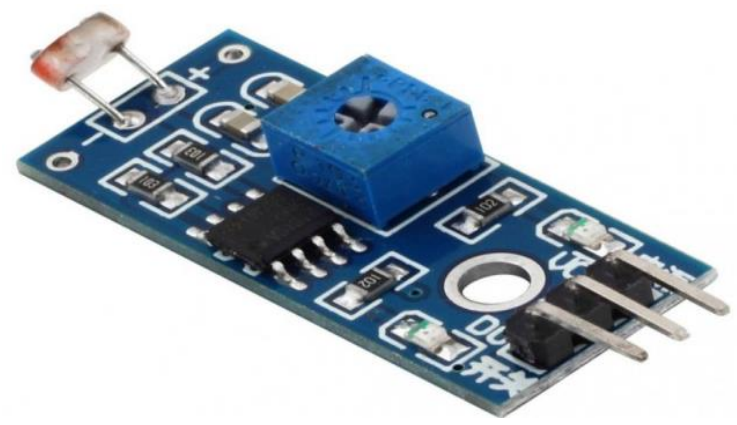

Fig. 6: LDR sensor

\section{Specifications:}

1. The input voltage can be applied 3.3-5V DC.

2. The output will be in both analog and digital.

3. Sensitivity will be adjusted.

PIR sensor: PIRs are made of piezoelectric sensors. In the center, there is a round metal can with a rectangular crystal that detects IR radiation levels. Everything emits low-level radiation, and if there is something hotter than the emission of radiation will be more. The PIR sensor is shown in figure 7. Motion detector has a sensor and it is divided into two halves. This is to detect motion (change) and not average IR levels. Those two are alied so they cancel out both. The output will swing high or low when one-half sees high or low IR radiations than the other.

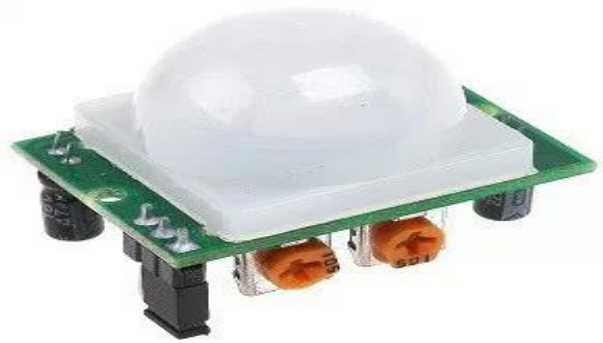

Fig. 7: PIR sensor

Arduino: NodeMCU Dev Board is based on extensively examined esp8266 SoC from Express if. It has integrated characteristics of WIFI access point and station + microcontroller and utilizes easy programming language based on LUA ESP8266. NodeMCU offers-

1. It is purely advanced Arduino -like hardware IO

2. The network applications will be as Event-driven API

3. Wi-Fi networking in order to connect to internet to fetch or upload data.

Raspberry Pi 3: The third generation Raspberry Pi is Raspberry Pi 3 Model B. It is just a board like credit card sized computer which can be used for many applications and replaces the original Raspberry Pi Model B and Pi 2 Model B. It is more than five times faster than the past two generations of Raspberry Pi board. In addition to this, it adjoins wireless LAN and Bluetooth connectivity which makes an ideal solution for powerful connected designs.

Internet: HTTP protocol has a wide usability in IoT platform. This communication provides reliability. The figure 7 tells that how the devices and user will be interacted. It is an important protocol among all the available protocols for accessing the internet.

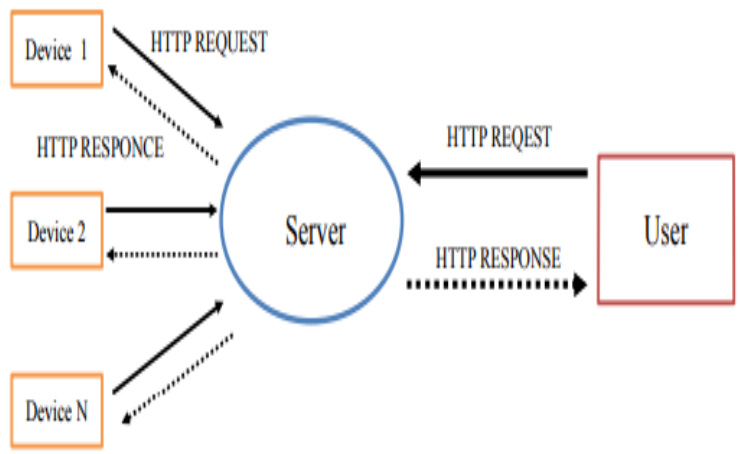

Fig. 8: System configuration using HTTP

The sequence diagram of HTTP communication is shown in figure 8. Tiny packets have to be transferred in large extent.

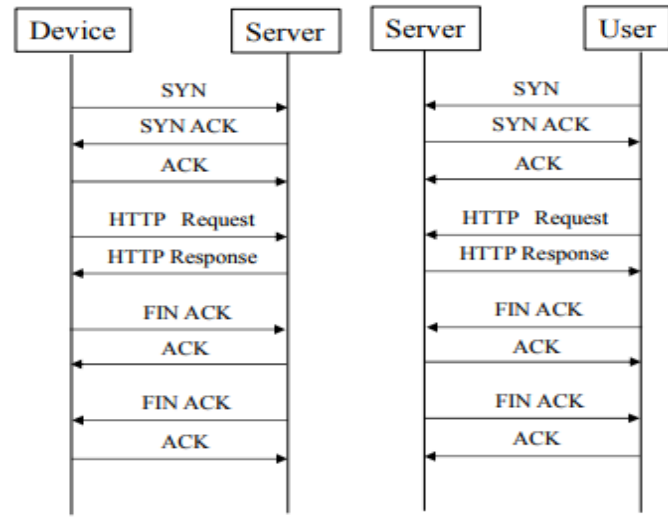

Fig. 8: Communication sequences of HTTP 


\section{Results}

PWM output in oscilloscope is shown in figure 9. PWM pulses are generated as per the commands received by it.

gital oxil casp:

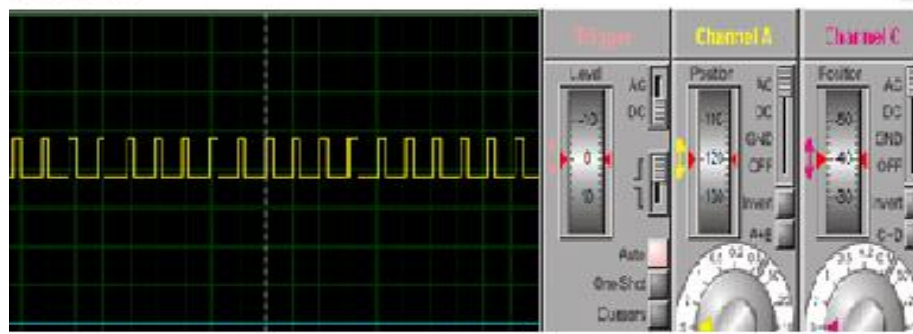

italCscilcscope
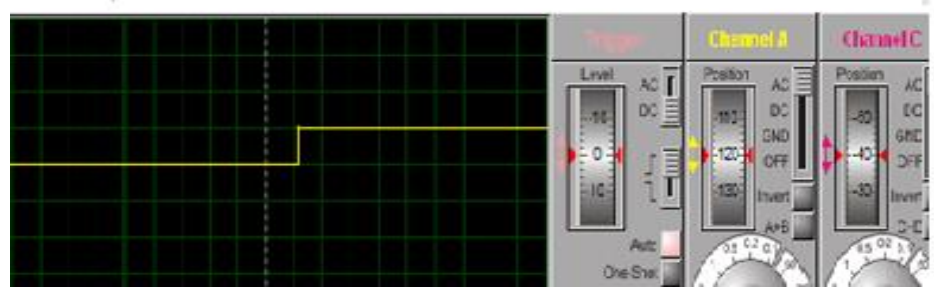
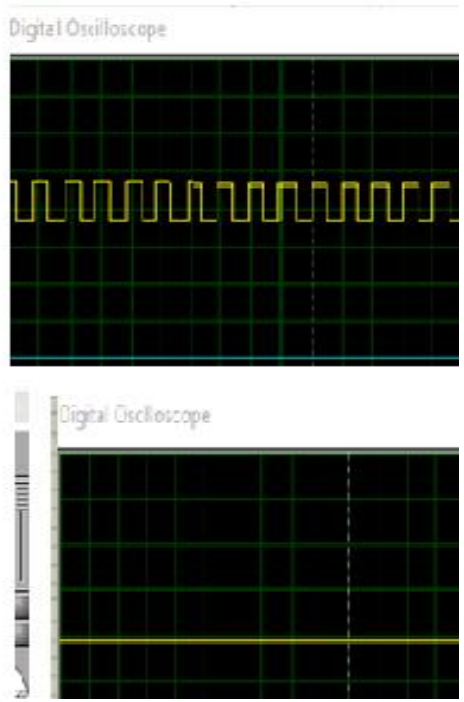

Fig. 9: PWM output in Oscilloscope

Power and energy consumption values are shown in figure 10.

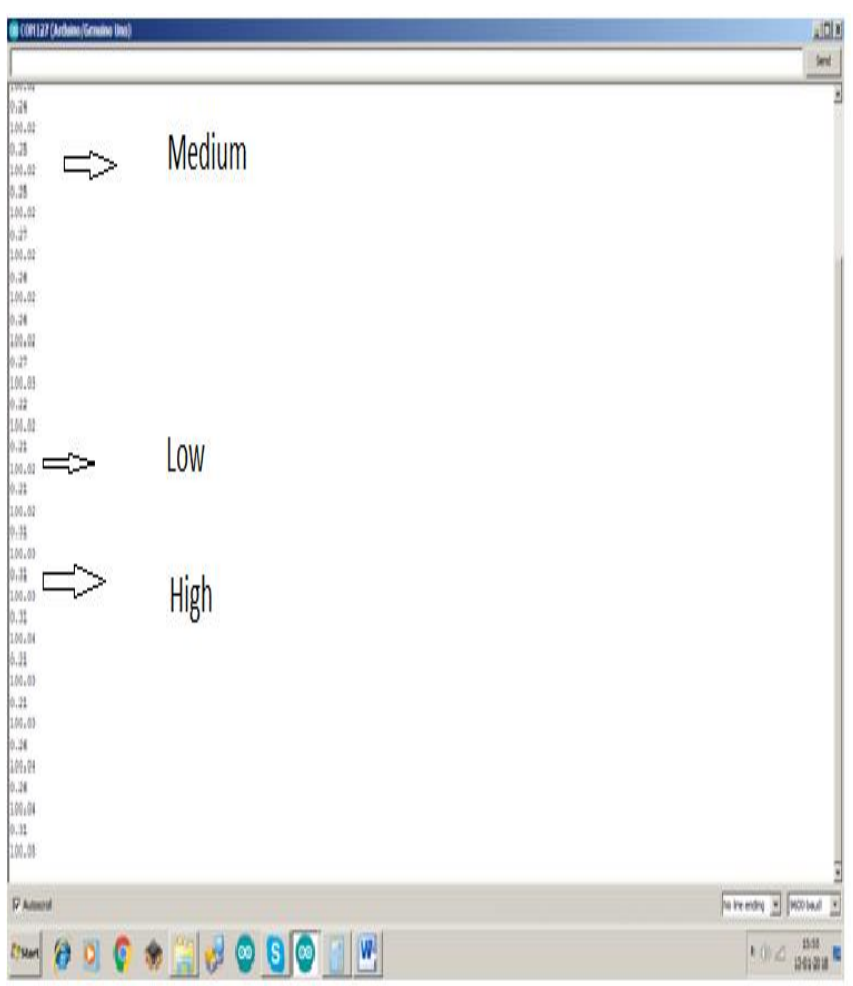

Fig. 10: Power and energy consumption values at different (low, medium high intensity levels)

The bar graph shown in below figure 11 explains that the difference between the power consumption of the existing street lighting system and the dimmer based proposed street lighting system. The testing has been done in two methods that is, with intensity reduction by changing the voltage and without intensity change. Also by using different wattage lamps the circuit has been tested. The dimmer based Street lighting system has consumed less power compared to existing system.

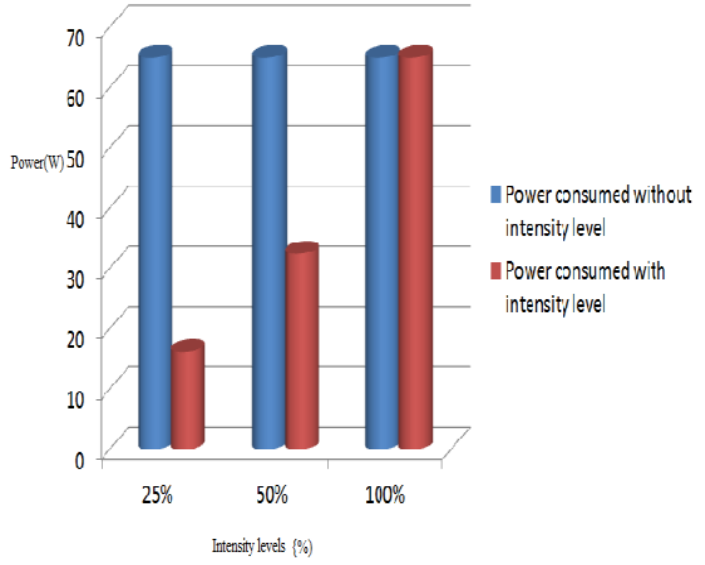

Fig. 11: Power consumption difference between existing and the dimmer based proposed street lighting systems

\section{Conclusion and Future Work}

IoT based smart street lighting system project has been designed in proteus software with two slave nodes and one master node. As per our requirement the Light glows. Intensity of light is reduced with dimmer circuit. The power consumption and energy consumption has been calculated and shown as output. Proposed design will be implementing using hardware. The output results will be uploaded to cloud. According to those results the government, offices and educational institutions will get benefit .Hence the Proposed IoT based smart street lighting system will give good results as per the requirement.

\section{References}

[1] C. Jane, G., Su, C.-C., Chiu, H.-J., and Lo, Y.-K. (2012). "Highefficiency led driver for street light applications." 2012 International Conference on Renewable Energy Research and Applications (ICRERA), 1-5 (Nov).

[2] Cacciatore, G. Fiandrino, C, Kliazovich, D, Granelli, F., and Bouvry, P. (2017). "Cost analysis of smart lighting solutions for smart cities." 2017 IEEE International Conference on Communications (ICC), 1-6 (May).

[3] Tetsuya Yokotani , Yuya Sasak," Comparison with HTTP and MQTT on Required Network Resources for IoT" The 2016 
International Conference on Control, Electronics, Renewable Energy and Communications (ICCEREC), 978-1-5090-07448/16/\$31.00 @2016 IEEE

[4] Charalampidis, P., Tragos, E., and Fragkiadakis, A. (2017). “A fog-enabled iot platform for efficient management and data collection." 2017 IEEE 22nd International Workshop on Computer Aided Modeling and Design of Communication Links and Networks (CAMAD), 1-6 (June).

[5] Dai, R. B., Turhan, M., YÄ'sldÄ'srÄ'sm, D., and UÃ gak, C. (2013). "A dimmer circuit for various lighting devices." 2013 8th International Conference on Electrical and Electronics Engineering (ELECO), 278-282 (Nov).

[6] Mahoor, M., Salmasi, F. R., and Najafabadi, T. A. (2017). "A hierarchical smart street lighting system with brute-force energy optimization.” IEEE Sensors Journal, 17(9), 2871-2879.

[7] Shere, V. and Suryawanshi, R. (2016). "A review on triac controlled energy efficient dimmable led driver for street lighting." 2016 International Conference on Automatic Control and Dynamic Optimization Techniques (ICACDOT), 239-241 (Sept).

[8] Tang, B., Chen, Z., Hefferman, G., Pei, S., Wei, T., He, H., and Yang, Q. (2017). "Incorporating intelligence in fog computing for big data analysis in smart cities." IEEE Transactions on Industrial Informatics, 13(5), 2140-2150. 21

[9] Akash RB, Holabasappa K, Kiran Kumar DM , Kiran Mardi, Asst Prof Smt.B.M.Nandini, "STREET LIGHT MONITORING AND CONTROLLING SYSTEM", International Journal of Modern Trends in Engineering and Research (IJMTER) Volume 02, Issue 04, [April 2015]

[10] Abdul Latif Saleem, Raja Sagar R, Sachin Datta N S, Sachin H S, Usha M S,"Street Light Monitoring and Control System", International Journal of Engineering and Techniques - Volume 1 Issue2, Mar Apr 2015

[11] David r. Gozzard , Sascha w. Schediwy, benjamin courtneybarrer, richard whitaker, and keith grainge, "simple stabilized radio-frequency transfer with optical phase actuation" ieee photonics technology letters, vol. 30, no. 3, february 1, 2018

[12] Fadi al-turjman 1 , Yoney kirsal ever2 , enver ever 1 , huan $\mathrm{x}$ Nguyen3, and deebak bakkiam david1 "seamless key agreement framework for mobile-sink in iot based cloud-centric secured public safety sensor networks" special section on mission critical public-safety communications: architectures, enabling technologies, and future applications volume 5, 2017, october 25 , 2017

[13] Wen-Long Chin, Wan Li, and Hsiao-Hwa Chen "Energy Big Data Security Threats in IoT-Based Smart Grid Communications" IEEE Communications Magazine, October 2017

[14] Badis Hammi, Rida Khatoun," IoT technologies for smart cities" IET Netw., 2018, Vol. 7 Iss. 1, pp. 1-13 (C) The Institution of Engineering and Technology 2017

[15] S.V.Manikanthan and K.Baskaran "Low Cost VLSI Design Implementation of Sorting Network for ACSFD in Wireles Sensor Network", CiiT International Journal of Programmable Device Circuits and Systems,Print: ISSN 0974 - 973X \& Online: ISSN 0974 - 9624, Issue : November 2011, PDCS112011008.

[16] T. Padmapriya and V. Saminadan, "Distributed Load Balancing for Multiuser Multi-class Traffic in MIMO LTE-Advanced Networks", Research Journal of Applied Sciences, Engineering and Technology (RJASET) - Maxwell Scientific Organization , ISSN: 2040-7459; e-ISSN: 2040-7467, vol.12, no.8, pp:813-822, April 2016 